Public reporting burden for this collection of information is estimated to average I hour per response, including the time for reviewing instructions, searching existing data sourcer. gathering and maintaining the data needed. and completing and reviewing the coliection of information. Send comments regarding this burden estimate or any other aspect of this collection of information, induding suggestions for reducing this burden, to Washington Headquarters services. Directorate lor lnf Davis Highway, Sulte 1204, Arlington, VA 22202-4302, and to the Office of Management and Budget, Paperwork Reduction Project (0204-0188), Washington, OC 20s03.

1. AGENCY USE ONLY (Leave blank) 1997 4. TITLE AND SUBTITLE

Matrix methods for population analysis

6. AUTHOR(S)

Hal Caswell

7. PERFORMING ORGANIZATION NAME(S) AND ADDRESS(ES)

WOODS HOLE OCEANOGRAPHIC INSTITUTION

WOODS HOLE, MA 02543

9. SPONSORING/MONITORING AGENCY NAME(S) AND ADDRESS(ES)

OFFICE OF NAVAL RESEARCH

ENVIRONMENTAL SCIENCES

DIRECTORATE

ARL INGTON, VA 22217-5660

\section{REPORT TYPE AND DATES COVERED} TECHNICAL

11. SUPPLEMENTARY NOTES

In citing this report in a bibliography, the reference given should be:

In: S. Tuljapurker and H. Caswell. 1997. Structured-population models in marine, terrestrial

and freshwater systems. Chapman and Hall, New York.

12a. DISTRIBUTION/AVAILABILITY STATEMENT

12b. OISTRIBUTION CODE

APPROVED FOR PUBLIC RELEASE:

DISTRIBUTION UNLIMITED

13. ABSTRACT (Maximum 200 words)

$$
\text { none }
$$

NSF DEb 92-11945

NSF OCE 93-02874

ONR N00014-92-J-1527

8. PERFORMING ORGANIZATION REPORT NUMBER

WHOI CONTR. 9224

10. SPONSORING/MONITORING AGENCY REPORT NUMBER

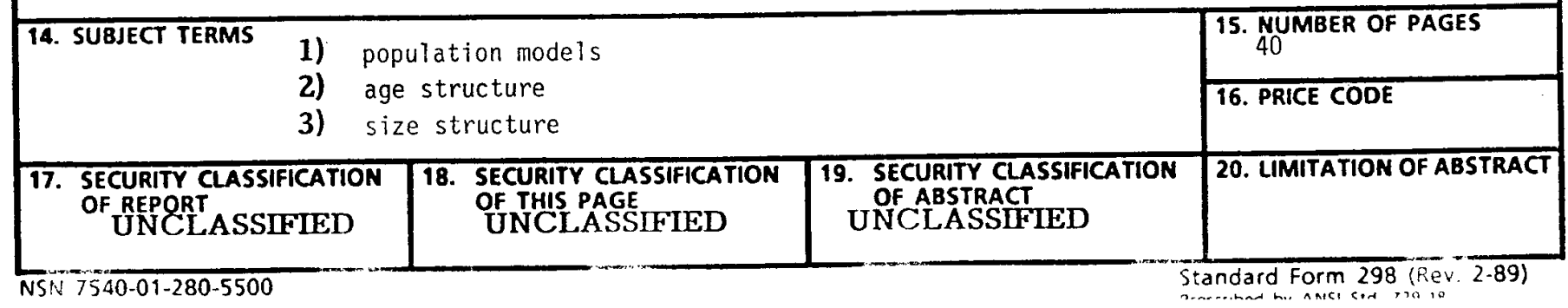


S. Tuljapurkar and H. Caswell. 1997. Structured-Population Models in Marine, Terrestrial and Freshwater Systems. Chapman and Hall, New York.

\title{
Matrix Methods for Population Analysis
}

\author{
Hal Caswell
}

Matrix models for structured populations were introduced by P. H. Leslie in the 1940's (Leslie 1945, 1948). Although they are in some ways the simplest of the mathematical approaches to structured population modeling (see Chapter 1), their analysis requires computational power. For this reason, and because ecologists of the day viewed matrix algebra as an esoteric branch of advanced mathematics, they were largely neglected until the late 1960's, when they were rediscovered by ecologists (Lefkovitch 1965) and human demographers (Goodman 1967; Keyfitz 1967). In the 1970's, matrix models were adopted by plant ecologists, who discovered that they could easily handle the complexity of plant life cycles in which size or developmental stage was more important than chronological age in determining the fate of individuals (Sarukhán \& Gadgil 1974; Hartshorn 1975; Werner \& Caswell 1977).

This chapter introduces the construction and analysis of matrix population models. I will not try to be comprehensive; I have done that elsewhere in book form (Caswell 1989a) and twice in simplified form with a focus on particular taxa (Caswell 1986; McDonald \& Caswell 1993). Instead, I try to convey the basics of matrix population models clearly and briefly. Wherever possible, I use different derivations than before (Caswell 1989a), so you may find some new ways to understand the source of some familiar results. I rarely cite my book (Caswell 1989a) (in spite of having done so three times in this paragraph); almost every topic presented here could be followed by the instruction, "see the book for more

[DITC QDALITY INTSEEUTED 
information." My focus here is on methods; I am sparing in my use of examples, because they can be found in many of the other chapters in this volume.

A note about notation. I use boldface symbols to denote vectors (lower case, as in $\mathbf{n}$ ) and matrices (uppercase, as in $\mathbf{A}$ ). Entries of vectors and matrices are lowercase letters with subscripts, so the $i$ th entry of $\mathbf{n}$ is $n_{i}$, and the element in the $i$ th row and $j$ th column of $\mathbf{A}$ is $a_{i j}$. Sometimes I use parenthetical superscripts to label matrices or vectors. Thus, $\mathbf{A}_{m}$ or $\mathbf{A}^{(m)}$ might both be used to denote the $m$ th in a series of matrices; the $i j$ th element of this matrix is written $a_{i j}^{(m)}$. The transpose of the matrix $\mathbf{A}$ is $\mathbf{A}^{\mathrm{T}}$. If $x=a+b i$ is a complex number, the complex conjugate is denoted by $\bar{x}=a-b i$. The complex-conjugate transpose of $\mathbf{A}$ is $\mathbf{A}^{*}$. The scalar product of two vectors is $\langle\mathbf{x}, \mathbf{y}\rangle=\mathbf{y}^{*} \mathbf{x}$.

\section{Formulating Matrix Models}

A matrix population model operates in discrete time, projecting a population from $t$ to $t+1$. The first step in formulating a matrix model is to define the time scale for the projection; this is called the projection interval. Models for the same population with different projection intervals may look quite different.

The second step is to choose a set of state variables for individuals ( $i$-state variables); these provide the information necessary to determine the response of an individual to the environment, over a projection interval. Examples of $i$-state variables include age, size, developmental stage, and geographical location.

A matrix model uses discrete stages, so the third step is to define a set of discrete categories for each $i$-state variable. Some $i$-states are naturally discrete (e.g., instars), while others are naturally continuous and must be made discrete (e.g., size). Dividing continuous variables into discrete categories involves trade-offs. A model treats all individuals within a category as identical, so creating only a few large categories reduces the accuracy of the $i$-state dynamics. Creating many small categories, alternatively, leads to a large model and may make it hard to estimate parameter values because sample sizes in each category are small.

The stages describe the life cycle, or as much of it as we believe to be demographically important. The next step is to translate them into a model. The life-cycle graph is a useful tool for this translation. 
The Life-Cycle Graph

A life-cycle graph describes the transitions an individual can make, during a projection interval, among the $i$-state categories that define its life cycle. To construct the graph, first draw a numbered point (a "node" in graph-theory terminology) for each $i$-state category. If, for example, size is the $i$-state variable, then the life-cycle graph contains a node for each size class. If age and size are both $i$-state variables, then the life-cycle graph contains a node for each age-size category. Draw arrows, "directed arcs," between nodes to indicate where it is possible for an individual in one stage to contribute individuals to another stage over a single projection inter$\mathrm{val}$. The head of the arrow shows the direction in which individuals move. If individuals can contribute in both directions between two stages, draw two arrows, rather than an arrow with a head on both ends. Contributions from one stage to another can result from the movement of individuals from one stage to another (e.g., by growth or aging) or from production of new individuals (e.g., by birth).

With each arrow is associated a coefficient; the coefficient on the arrow from stage $j$ to stage $i$ is denoted $a_{i j}$ (the ordering of the subscripts is important; it corresponds to the arrangement of coefficients in the resulting matrix model). The coefficient $a_{i j}$ gives the number of stage $i$ individuals at $t+1$ per stage $j$ individual at time $t$.

So far, we have made no decisions about the nature of these coefficients; I return to this below.

Figure $1 a$ shows a life-cycle graph for an age-classified model with the age interval equal to the projection interval. Individuals in one age class can contribute to another only by surviving to the next older age class or by reproduction to the first age class. Figure $1 b$ shows the graph for a size-classified model in which individuals may grow to the next size class, remain in their own size class, and possibly reproduce new individuals in the first size class. Suppose some individuals in the first size class grow so rapidly that after one projection interval they are in the third size class. This would require the modification shown in Figure $c, 1$ where an arrow has been drawn from stage 1 to stage 3 .

The interpretation of the coefficients depends on the identity of the stages and the processes involved in the transitions. In Figure $1 a$, the $P_{i}$ are age-specific survival probabilities and the $F_{i}$ are age-specific fertilities. In Figures $1 b$ and $1 c$, the $G_{i}$ are probabilities of surviving and growing, the $P_{i}$ are probabilities of surviving 
(a)

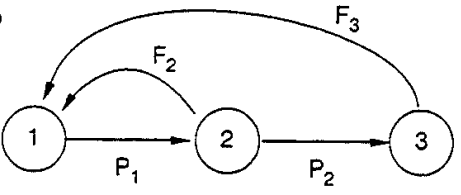

(b)
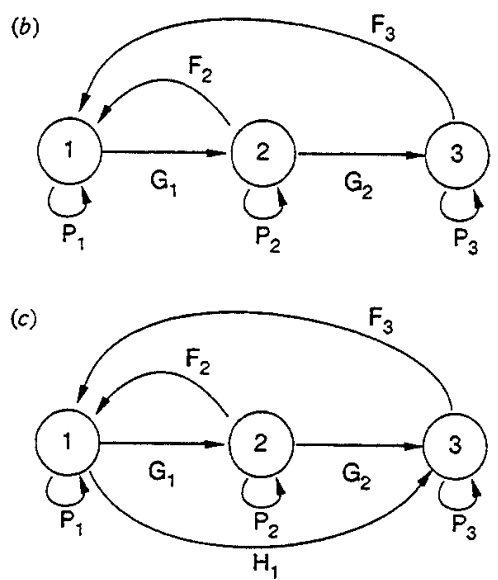

FIGURE 1. Three life-cycle graphs. (a) An age-classifed model with three age classes; the $P_{i}$ are age-specific survival probabilities, and the $F_{i}$ are age-specific fertilities. (b) A size-classified model with three size classes; the $G_{i}$ are size-specific probabilities of survival and growth, the $P_{i}$ are size-specific probabilities of surviving and remaining in the same size class, and the $F_{i}$ are size-specific fertilities. (c) The same life-cycle graph as in (b), but with an additional transition ( $\left.H_{1}\right)$ from size class 1 to size class 3.

and not growing, and the $F_{i}$ are size-specific fertilities. In Figure 1c, the coefficient $H_{1}$ is the probability of surviving and growing enough to move from size class 1 to size class 3. Demographers use the term vital rates to refer collectively to the rates of survival, growth, reproduction, and any other important demographic processes.

\section{A Set of Difference Equations}

The life-cycle graph corresponds directly to a model written as a set of difference equations. For the size-classified graph in Figure 1b, remembering the definitions of the coefficients, the set of equations 
describing the population is

$$
\begin{aligned}
& n_{1}(t+1)=P_{1} n_{1}(t)+F_{2} n_{2}(t)+F_{3} n_{3}(t) \\
& n_{2}(t+1)=G_{1} n_{1}(t)+P_{2} n_{2}(t) \\
& n_{3}(t+1)=G_{2} n_{2}(t)+P_{3} n_{3}(t) .
\end{aligned}
$$

It is worth looking at these equations for a moment. The first states that the number of individuals in stage 1 at $t+1$ is the sum of those remaining in stage 1 from time $t$ and those contributed by reproduction from stages 2 and 3 . The second equation states that the number in stage 2 at $t+1$ is the sum of those growing into stage 2 from stage 1 and those remaining in stage 2 from time $t$. The third equation says the same thing for stage 3 .

The equations corresponding to Figure $1 c$ are

$$
\begin{array}{rr}
n_{1}(t+1)= & P_{1} n_{1}(t)+F_{2} n_{2}(t)+F_{3} n_{3}(t), \\
n_{2}(t+1)= & G_{1} n_{1}(t)+P_{2} n_{2}(t), \\
n_{3}(t+1)= & H_{1} n_{1}(t)+G_{2} n_{2}(t)+P_{3} n_{3}(t) .
\end{array}
$$

It would be possible to write down these equations directly, without using the life-cycle graph, if we were clear about the nature of the possible transitions, which in turn depends on the definition of the stages. But using the life-cycle graph makes it easier, and helps to guard against mistakes in defining the stages and transitions.

\section{The Matrix Model}

The system of difference equations derived from the life-cycle graph can be written more simply in matrix form:

$$
\mathbf{n}(t+1)=\mathbf{A} \mathbf{n}(t)
$$

where

$$
\mathbf{n}(t)=\left[\begin{array}{c}
n_{1}(t) \\
n_{2}(t) \\
\vdots \\
n_{k}(t)
\end{array}\right]
$$

is a stage-distribution vector and $\mathbf{A}$ is a population-projection matrix. The elements of this matrix can be obtained from the system of difference equations or directly from the life-cycle graph: the $i j$ th entry of $\mathbf{A}$ is the coefficient on the arrow from stage $j$ to 
stage $i$. The reason for the order of the subscripts is to guarantee this correspondence.

Applying this rule to the life-cycle graphs in Figure 1 yields

$$
\begin{aligned}
\mathbf{A}^{(a)} & =\left[\begin{array}{ccc}
0 & F_{2} & F_{3} \\
P_{1} & 0 & 0 \\
0 & P_{2} & 0
\end{array}\right], \\
\mathbf{A}^{(b)} & =\left[\begin{array}{ccc}
P_{1} & F_{2} & F_{3} \\
G_{1} & P_{2} & 0 \\
0 & G_{2} & P_{3}
\end{array}\right], \\
\mathbf{A}^{(c)} & =\left[\begin{array}{ccc}
P_{1} & F_{2} & F_{3} \\
G_{1} & P_{2} & 0 \\
H_{1} & G_{2} & P_{3}
\end{array}\right] .
\end{aligned}
$$

The age-classified model produces a special matrix, with positive entries only on the first row (fertilities) and the subdiagonal (survival probabilities). Such a matrix is often called a Leslie matrix, in recognition of the early papers of Leslie $(1945,1948)$.

I have said nothing about how the numerical values of the coeffcients $a_{i j}$ are obtained. This obviously important question deserves its own chapter (see Chapter 19, by Wood, for one approach), but here I assume that the matrix is at hand and focus on how to analyze it.

\section{Types of Matrix Models}

The coefficient $a_{i j}$ is the contribution of each individual in stage $j$ to the number of individuals in stage $i$ during one projection interval. What happens in the next projection interval? Depending on the answer to this question, matrix models fall into three classs, each with its own analytical approach.

Linear, constant-coefficient models. If the coefficients $a_{i j}$ are constants, the resulting model is linear and time-invariant:

$$
\mathbf{n}(t+1)=\mathbf{A n}(t) .
$$

This is the simplest case; it can be analyzed in great detail, and it is widely used. But in reality the vital rates are not constants, so the biological interpretation of these results requires great care. 
Nonlinear models. If the $a_{i j}$ are not constant but depend on the current state of the population, the resulting model is nonlinear:

$$
\mathbf{n}(t+1)=\mathbf{A}_{n} \mathbf{n}(t)
$$

where $\mathbf{A}_{n}$ is the transition matrix evaluated at $\mathbf{n}$. The nonlinearity may result from density dependence (e.g., competition for resources), frequency dependence (e.g., competition for mates), or both.

Time-varying models. The coefficients may also change with time, independently of $\mathbf{n}(t)$. resulting in the model

$$
\mathbf{n}(t+1)=\mathbf{A}_{t} \mathbf{n}(t)
$$

D)werministic. periondic variation is often used to model seasonality or other kinds of cnvironmental periodicity. Alternatively, the cocfficients may vary stochastically, reflecting some random environmental process (see Chapter 3 , by Tuljapurkar). Time-varying models may be either linear or nonlinear.

\section{Objectives of Analysis}

The analysis of each of these types of model, although requiring different mathematical tools, addresses a set of similar questions. Imagine that you are in possession of a matrix population model. What you should do with it depends on the question you want to answer.

1. Transient analyses describe the short-term dynamics resulting from specific initial conditions.

2. Asymptotic analyses describe the long-term dynamics of the population.

(a) Population growth rate: what is the asymptotic rate of population growth or decline?

(b) Population structure: what are the relative abundances of the different stages in the life cycle?

(c) Ergodicity: are the dynamics, including the growth rate and the population structure, asymptotically independent of initial conditions?

(d) Attractors (mainly in density-dependent models): what are the qualitative properties of the asymptotic dynamics (fixed point, cycle, quasiperiodicity, chaos, etc.)? 
3. Perturbation analyses examine the effects of changes in parameter values or initial conditions on the results of the models. Three questions are of particular importance:

(a) Sensitivity and elasticity analysis of population growth rate: how does the growth rate respond to changes in vital rates?

(b) Stability analysis of equilibria: if initial conditions are perturbed slightly away from an equilibrium point, does the solution return to or depart from the neighborhood of the equilibrium?

(c) Bifurcation analysis: what happens to the asymptotic behavior of a nonlinear model as a parameter in the model is changed?

The methods used to address these questions depend on the nature and sometimes on the details of the model, but any populationmodeling project that does not address short-term dynamics, longterm dynamics, and the effects of perturbations on those dynamics has left something out.

\section{Analysis: The Linear Case}

We begin with the linear time-invariant model (6), in which $\mathbf{A}$ is a constant matrix. There are two justifications for spending time on this model, in spite of the fact that the vital rates of any real population are certainly not constant. The first is theoretical: understanding population dynamics in the simplest case is a fundamental step in understanding more-complicated cases. The second is practical: when interpreted as a projection rather than a prediction (Keyfitz 1968; Caswell 1989a), the results of a linear model provide a valuable characterization of the current environment by calculating the purely hypothetical consequences of maintaining that environment forever. Linear matrix population models are frequently used in this way, as a form of demographic analysis of vital-rate data, rather than as a prediction of future population dynamics.

\section{Exponential Solutions and the Characteristic Equation}

One approach to equation (6) is to conjecture that, like other linear equations, it has an exponential solution(s),

$$
\mathbf{n}(t)=\lambda^{t} \mathbf{w}
$$


for some fixed vector $\mathbf{w}$. Substituting this into (6) gives

$$
\lambda^{t+1} \mathbf{w}=\lambda^{t} \mathbf{A} \mathbf{w} \text {. }
$$

A scalar $\lambda$ and a vector $\mathbf{w}$ that satisfy this relation are called an eigenvalue and eigenvector of $\mathbf{A}$, respectively. They must satisfy

$$
(\mathbf{A}-\lambda \mathbf{I}) \mathbf{w}=0,
$$

which has a nonzero solution for $\mathbf{w}$ only if the determinant of the matrix $\mathbf{A}-\lambda \mathbf{I}$ equals zero. This is called the characteristic equation:

$$
\operatorname{det}(\mathbf{A}-\lambda \mathbf{I})=0 \text {. }
$$

\section{The Spectral Decomposition of $\mathbf{A}$}

An alternative approach is to begin by solving equation (6), starting from a specified initial population $\mathbf{n}\left(t_{0}\right)$. By repeatedly applying (6), we see that $\mathbf{n}\left(t_{0}+1\right)=\mathbf{A} \mathbf{n}\left(t_{0}\right), \mathbf{n}\left(t_{0}+2\right)=\mathbf{A}^{2} \mathbf{n}\left(t_{0}\right)$, and in general

$$
\mathbf{n}\left(t_{0}+t\right)=\mathbf{A}^{t} \mathbf{n}\left(t_{0}\right)
$$

Thus, to understand population dynamics over time we need only understand the behavior of $\mathbf{A}^{t}$.

One approach to the problem is via the spectral decomposition of $\mathbf{A}$, which makes it possible to evaluate any function of $\mathbf{A}$, including $\mathbf{A}^{t}$. First, note a few facts about the eigenvalues and eigenvectors of a matrix. The vectors $\mathbf{w}$ and $\mathbf{v}$ are right and left eigenvectors of $\mathbf{A}$ if there is a (possibly complex) scalar $\lambda$ such that

$$
\begin{aligned}
\mathbf{A} \mathbf{w} & =\lambda \mathbf{w}, \\
\mathbf{v}^{*} \mathbf{A} & =\lambda \mathbf{w}^{*},
\end{aligned}
$$

where the asterisk denotes the complex-conjugate transpose. A left eigenvector $\mathbf{v}$ of $\mathbf{A}$, corresponding to $\lambda$, is a right eigenvector of $\mathbf{A}^{*}$ corresponding to $\bar{\lambda}$; that is,

$$
\mathbf{A}^{*} \mathbf{v}=\bar{\lambda} \mathbf{v}
$$

The eigenvalues are found as the solutions of the characteristic equation (10).

If $\mathbf{A}$ is a $k \times k$ matrix, the characteristic equation is a polynomial of degree $k$ and has $k$ solutions $\lambda_{i}, i=1,2, \ldots, k$. The corresponding eigenvectors are $\mathbf{w}_{i}$ and $\mathbf{v}_{i}, i=1,2, \ldots, k$. I assume that these eigenvalues are all distinct, as seems to be true in practice for population-projection matrices. This assumption guarantees that 
the right eigenvectors and left eigenvectors, respectively, are linearly independent sets.

Let $\langle\mathbf{w}, \mathbf{v}\rangle=\mathbf{v}^{*} \mathbf{w}$ denote the scalar product of $\mathbf{w}$ and $\mathbf{v}$. The left and right eigenvectors can always be scaled so that $\left\langle\mathbf{v}_{i}, \mathbf{w}_{i}\right\rangle=1$. In addition, the left and right eigenvectors corresponding to different eigenvalues are orthogonal, so that $\left\langle\mathbf{v}_{i}, \mathbf{w}_{j}\right\rangle=0$ if $i \neq j$.

Any matrix $\mathbf{A}$ with distinct eigenvalues can be written in the form

$$
\mathbf{A}=\lambda_{1} \mathbf{Z}_{1}+\cdots+\lambda_{k} \mathbf{Z}_{k},
$$

where the matrices $\mathbf{Z}_{i}$, known as the constituent matrices of $\mathbf{A}$, are given by

$$
\mathbf{Z}_{i}=\mathbf{w}_{i} \mathbf{v}_{i}^{*} .
$$

That is, $\mathbf{Z}_{i}$ is a matrix whose columns are all proportional to $\mathbf{w}_{i}$ and whose rows are all proportional to $\mathbf{v}_{i}^{*}$.

The constituent matrices have two important properties. First,

$$
\begin{aligned}
\mathbf{Z}_{i}^{2} & =\mathbf{w}_{i} \mathbf{v}_{i}^{*} \mathbf{w}_{i} \mathbf{v}_{i}^{*} \\
& =\mathbf{w}_{i}\left\langle\mathbf{w}_{i}, \mathbf{v}_{i}\right\rangle \mathbf{v}_{i}^{*} \\
& =\mathbf{Z}_{i} .
\end{aligned}
$$

(Such matrices are called idempotent.) Second, multiplying two different constituent matrices yields a zero matrix:

$$
\begin{aligned}
\mathbf{Z}_{i} \mathbf{Z}_{j} & =\mathbf{w}_{i} \mathbf{v}_{i}^{*} \mathbf{w}_{j} \mathbf{v}_{j}^{*} \\
& =\mathbf{w}_{i}\left\langle\mathbf{w}_{j}, \mathbf{v}_{i}\right\rangle \mathbf{v}_{j}^{*} \\
& =\mathbf{0} .
\end{aligned}
$$

These properties are useful because, together, they imply that

$$
\mathbf{A}^{2}=\left(\sum_{i} \lambda_{i} \mathbf{Z}_{i}\right)\left(\sum_{j} \lambda_{j} \mathbf{Z}_{j}\right)=\sum_{i} \lambda_{i}^{2} \mathbf{Z}_{i}
$$

Multiplying repeatedly by $\mathbf{A}$, it is not hard to see that

$$
\mathbf{A}^{t}=\sum_{i} \lambda^{t} \mathbf{Z}_{i}
$$

This result, together with equation (13), yields our desired expression for the dynamics of a population described by (8):

$$
\mathbf{n}\left(t_{0}+t\right)=\sum_{i} \lambda_{i}^{t} \mathbf{Z}_{i} \mathbf{n}\left(t_{0}\right)
$$


The only parts of the right-hand side of (20) that vary with time are the factors $\lambda_{i}^{t}$. The behavior of $\lambda_{i}^{t}$ depends on the sign of $\lambda_{i}$ and on whether $\lambda_{i}$ is real or complex. If $\lambda_{i}$ is real and positive, $\lambda_{i}^{t}$ grows or decays exponentially, depending on whether $\lambda_{i}$ is greater or less than one. If $\lambda_{i}$ is real and negative, $\lambda_{i}^{t}$ oscillates between positive and negative values, growing or decaying in magnitude depending on whether $\left|\lambda_{i}\right|$ is greater or less than one. If $\lambda_{i}$ is complex, $\lambda_{i}^{t}$ oscillates in a sinusoidal pattern, growing or decaying in magnitude depending on whether $\left|\lambda_{i}\right|$ is greater or less than one.

Since the dynamic properties of the population are determined by the eigenvalues of $\mathbf{A}$, it behooves us to see what we can say, a priori and in general, about these eigenvalues.

\section{Eigenvalues, Eigenvectors, and the Perron-Frobenius Theorem}

We can safely assume that the elements of $\mathbf{A}$ are nonnegative. Negative elements in $\mathbf{A}$ imply the possibility of negative individuals, which I prefer not to deal with. Perhaps surprisingly, this simple assumption tells us almost everything we want to know about the eigenvalues and eigenvectors of $\mathbf{A}$, thanks to a mathematical result known as the Perron-Frobenius theorem. In order to state the theorem, we need two more properties of A: irreducibility and primitivity.

A matrix $\mathbf{A}$ is irreducible if and only if its life-cycle graph is connected, that is, if there is a path, following the direction of the arrows, from every stage to every other stage. A matrix $\mathbf{A}$ is primitive if and only if there is some integer $k$ such that every element of $\mathbf{A}^{k}$ is strictly greater than zero. A more biologically revealing criterion is based on the life-cycle graph. Define a loop as a sequence of arrows, traversed in the direction of the arrows, that begins and ends at the same node, without passing through any node twice. The matrix $\mathbf{A}$ is primitive if and only if the greatest common divisor of the lengths of the loops in the life-cycle graph is one. Any primitive matrix is also irreducible. Most populationprojection matrices encountered in practice are both irreducible and primitive.

What about matrices that are reducible or imprimitive (i.e., not primitive)? A reducible matrix has some stages that make no contribution to some other stages; the life-cycle graph breaks into two (or more) pieces with only one-way communication. The most common example is a life cycle with post-reproductive stages; from 


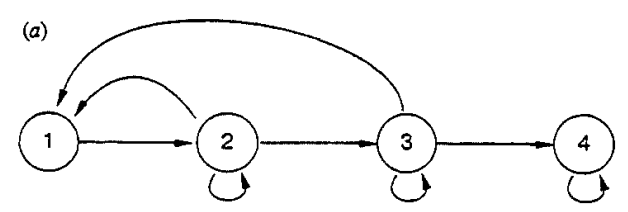

(b)

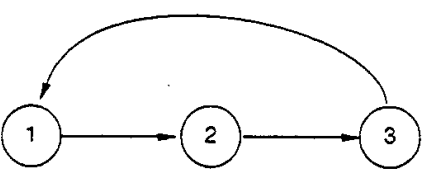

Figure 2. (a) A life-cycle graph corresponding to a reducible matrix. Stage 4 represents post-reproductive females; there is no pathway from this stage to any of the earlier stages. (b) A life-cycle graph corresponding to an imprimitive matrix. This is a semelparous age-classified model; individuals that survive to age class 3 reproduce and die.

such a stage there is no pathway back to the part of the life cycle that does reproduce. Figure $2 a$ shows an example; a graph of this form appears in a stage-classified model for killer whales (Brault \& Caswell 1993). An imprimitive life cycle has some underlying periodicity, so that the loops in the life-cycle graph are all multiples of some common loop length. Imprimitive matrices are sometimes called "cyclic" to reflect this fact. The most common example is a semelparous age-classified life cycle with a fixed age at reproduction (Fig. 2b). Only a single loop appears in such a life-cycle graph, with a length determined by the age at reproduction. Some kinds of seasonal models for annual organisms also produce imprimitive matrices, reflecting the periodicity imposed by the annual cycle of the seasons. The graphs in Figure 2 contain no coefficients because reducibility and primitivity depend on the form of the graph but not on the values of the coefficients.

The Perron-Frobenius theorem states that a nonnegative, irreducible, primitive matrix has three properties:

1. a simple (i.e., non-repeated) eigenvalue $\lambda_{1}$ that is real, positive, and strictly greater in magnitude than any of its other eigenvalues,

2. a right eigenvector $w_{1}$ corresponding to $\lambda_{1}$, which is strictly positive (or can be made so by multiplying by a scalar) and is the only nonnegative right eigenvector, and

3. a left eigenvector $\mathbf{v}_{1}$ corresponding to $\lambda_{1}$, which is also strictly positive and is the only nonnegative left eigenvector. 
The Perron-Frobenius theorem also describes the eigenvalues and eigenvectors of imprimitive and reducible matrices (see Caswell $1989 a$ and, of course, many matrix texts, e.g., Seneta 1981; Horn \& Johnson 1985).

\section{Demographic Ergodicity}

The eigenvalue $\lambda_{1}$ (the dominant eigenvalue) plays a central role in the asymptotic analysis of linear matrix models. In equation (20), the growth of $\mathbf{n}\left(t_{0}+t\right)$ is given by a sum of terms involving the eigenvalues of $\mathbf{A}$ raised to higher and higher powers. Intuitively, as $t$ gets large, $\lambda_{1}^{t}$ increases more quickly, or decreases more slowly, than $\lambda_{i}^{t}$ for $i \neq 1$. Asymptotically, we expect the growth of the population to be determined by $\lambda_{1}$, whereas all the eigenvalues contribute to short-term transient behavior. More precisely,

$$
\begin{aligned}
\lim _{t \rightarrow \infty} \frac{\mathbf{n}\left(t_{0}+t\right)}{\lambda_{1}^{t}} & =\lim _{t \rightarrow \infty} \sum_{i}\left(\frac{\lambda_{i}}{\lambda_{1}}\right)^{t} \mathbf{Z}_{i} \mathbf{n}\left(t_{0}\right) \\
& =\mathbf{Z}_{1} \mathbf{n}\left(t_{0}\right) \\
& =\mathbf{w}_{1} \mathbf{v}_{1}^{*} \mathbf{n}\left(t_{0}\right) .
\end{aligned}
$$

This gives the following results on asymptotic dynamics, conditional on the primitivity of $\mathbf{A}$.

1. The population eventually grows geometrically at a rate given by $\lambda_{1}$ (the population growth rate or rate of increase).

2. Population structure eventually becomes proportional to $\mathbf{w}_{1}$ (the stable stage distribution).

3. The constant of proportionality relating population structure and $\mathbf{w}$ is a weighted sum of the initial numbers in each stage $\left(\mathbf{v}_{1}^{*} \mathbf{n}\left(t_{0}\right)\right)$. The weights are the elements in $\mathbf{v}_{\mathbf{1}}$; the vector $\mathbf{v}_{\mathbf{1}}$ thus gives the relative contributions of the stages to eventual population size (not population growth rate) and is called the reproductive-value vector.

The population eventually converges to the stable stage distribution, growing at a rate given by the dominant eigenvalue, regardless of the initial conditions (except, of course, the special case of a zero initial population). The property of forgetting the past and growing at a rate determined by the vital rates rather than by initial conditions is called ergodicity.

Because $\lambda_{1}, \mathbf{w}_{1}$, and $\mathbf{v}_{1}$ are properties of the vital rates rather than initial conditions, they are widely used as demographic statis- 
tics. They can provide valuable insight into the vital rates and the environmental conditions that determine them, but they cannot predict actual population dynamics; everyone "knows" that populations cannot grow geometrically forever. These statistics must be interpreted as projections of what would happen if the vital rates were to remain constant, rather than as predictions of what will happen. They characterize the present environment, not the future of the population.

Similar ergodic results hold for stochastic models (see Chapter 3) and density-dependent models. In each case, the asymptotic properties provide demographic statistics that are determined by the vital rates rather than by the historical accidents of initial conditions. They can be used just as $\lambda_{1}, \mathbf{w}_{1}$, and $\mathbf{v}_{1}$ are used in the linear case.

\section{Perturbation Analysis}

Only rarely are we interested in one precisely specified model. We can usually imagine that the model might change in some way, and would like to know how such changes would affect the results of the analysis. Perturbation analyses address this problem. In density-independent models, perturbation analyses focus on the eigenvalues and eigenvectors, whereas in density-dependent models perturbation analyses focus on the stability and bifurcation of equilibria.

A perturbation analysis of the eigenvalues of a population-projection matrix can answer several questions.

1. What are the effects of potential changes in the vital rates, as might result from strategies designed to protect endangered species (by: increasing $\lambda$ ) or control pest species (by reducing $\lambda)$ ?

2. Where should efforts to improve the estimates of the vital rates be focused in order to improve the accuracy of the estimate of $\lambda$ ? All else being equal, the biggest payoff comes from improving the estimates of the vital rates to which $\lambda$ is most sensitive, since errors in those estimates have the biggest effect.

3. Genetic variation produces individuals whose vital rates are perturbed from the overall population values; from these, natural selection chooses those perturbations whose carriers increase most rapidly. Which vital rates are under the greatest selective pressure? 
4. Suppose that some environmental differences (either natural or the result of experimental manipulation) have produced differences in the vital rates, and hence in $\lambda$, among two or more populations. How much do each of the vital-rate differences contribute to these observed differences in $\lambda$ ?

Fortunately, it is easy to calculate the sensitivity of $\lambda$ to a change in any of the vital rates, once we know the eigenvectors. The next subsection presents these calculations. Formulas also exist for the sensitivities of the eigenvectors $\mathbf{w}$ and $\mathbf{v}$, for the sensitivities of $\lambda$ for periodic time-varying models (Caswell \& Trevisan 1994), and for the sensitivities of the sensitivities themselves (Caswell 1996b).

\section{Sensitivity and Elasticity of Eigenvalues}

The sensitivity of population growth rate to changes in the vital rates can be calculated as the derivative of $\lambda$ to changes in the matrix elements $a_{i j}$. Suppose that $\lambda, \mathbf{w}$, and $\mathbf{v}$ satisfy

$$
\begin{aligned}
\mathbf{A} \mathbf{w} & =\lambda \mathbf{w} \\
\mathbf{v}^{*} \mathbf{A} & =\lambda \mathbf{v}^{*} \\
\langle\mathbf{w}, \mathbf{v}\rangle & =\mathbf{v}^{*} \mathbf{w}=1
\end{aligned}
$$

Now consider a perturbed matrix $\mathbf{A}+d \mathbf{A}$, where $d \mathbf{A}$ is a matrix of small perturbations $d a_{i j}$. The eigenvalues and eigenvectors of the new matrix satisfy

$$
(\mathbf{A}+d \mathbf{A})(\mathbf{w}+d \mathbf{w})=(\lambda+d \lambda)(\mathbf{w}+d \mathbf{w}) .
$$

Expanding the products and eliminating second-order terms yields

$$
\mathbf{A} \mathbf{w}+\mathbf{A}(d \mathbf{w})+(d \mathbf{A}) \mathbf{w}=\lambda \mathbf{w}+\lambda(d \mathbf{w})+(d \lambda) \mathbf{w},
$$

which simplifies to

$$
\mathbf{A}(d \mathbf{w})+(d \mathbf{A}) \mathbf{w}=\lambda(d \mathbf{w})+(d \lambda) \mathbf{w} .
$$

Multiplying both sides by $\mathbf{v}^{*}$ yields

$$
\mathbf{v}^{*} \mathbf{A}(d \mathbf{w})+\mathbf{v}^{*}(d \mathbf{A}) \mathbf{w}=\lambda \mathbf{v}^{*}(d \mathbf{w})+(d \lambda) \mathbf{v}^{*} \mathbf{w} .
$$

The first term on the left-hand side is the same as the first term on the right-hand side (because of eq. 23), and the last term on the right-hand side simplifies to $d \lambda$ (because of eq. 24), leaving

$$
\mathbf{v}^{*} d \mathbf{A} \mathbf{w}=d \lambda .
$$

If $d \mathbf{A}$ contains only a single nonzero element $d a_{i j}$, a change in only the $i j$ th element of $\mathbf{A}$, we obtain the fundamental sensitivity 
equation:

$$
\frac{\partial \lambda}{\partial a_{i j}}=\bar{v}_{i} w_{j}
$$

(The bar over $v_{i}$ has been ignored in most presentations of this formula, including mine. It is irrelevant to the case of the dominant eigenvalue of a population-projection matrix, which always has real eigenvectors, but it must be included for calculations involving other eigenvalues.)

Equation (30) says that the sensitivity of $\lambda$ to changes in $a_{i j}$ is proportional to the product of the reproductive value of stage $i$ and the representation of stage $j$ in the stable stage distribution.

The sensitivity of $\lambda$ to changes in other parameters can be calculated using the chain rule: for some parameter $x$,

$$
\frac{\partial \lambda}{\partial x}=\sum_{i, j} \frac{\partial \lambda}{\partial a_{i j}} \frac{\partial a_{i j}}{\partial x} .
$$

The sensitivity of $\lambda$ gives the effect of a small additive change in one of the vital rates. The effect of a small proportional change in a vital rate is given by the elasticity of $\lambda$ :

$$
e_{i j}=\frac{a_{i j}}{\lambda} \frac{\partial \lambda}{\partial a_{i j}} .
$$

In addition to giving the proportional change in $\lambda$ resulting from a proportional change in the $a_{i j}$, the elasticities also measure the contribution of the $a_{i j}$ to overall population growth rate. To be precise, $\sum_{i, j} e_{i j}=1$ (for a simple proof, see Mesterton-Gibbons 1993), and $e_{i j}$ can be interpreted as the proportion of $\lambda$ contributed by $a_{i j}$.

Elasticities to other parameters can also be calculated:

$$
\begin{aligned}
e(x) & =\frac{x}{\lambda} \frac{\partial \lambda}{\partial x} \\
& =\frac{x}{\lambda} \sum_{i, j} \frac{\partial \lambda}{\partial a_{i j}} \frac{\partial a_{i j}}{\partial x} .
\end{aligned}
$$

The elasticities of $\lambda$ with respect to other parameters do not in general sum to one, and they cannot be interpreted as contributions to population growth rate. 


\section{Sensitivity or Elasticity?}

Some authors seem to believe that sensitivities and elasticities are alternatives, that one is superior to the other, or that one or the other is biased in some way. This is not so; they provide accurate answers to different questions. The difference between them is comparable to the difference between plotting the same set of numbers on arithmetic (sensitivity) or logarithmic (elasticity) axes. Neither kind of graph is wrong, but one or the other may be better at revealing interesting patterns in the numbers. For more discussion, see Chapter 7 , by Horvitz et al.

\section{Life-Table-Response Experiments and Comparative Demography}

Life-table-response experiments (ITRE's) are manipulative experiments or comparative observations in which the dependent variable is a complete set of vital rates (loosely speaking, a life table; Caswell 1989b). The different environmental conditions (the "treatments") cause changes in the vital rates, which in turn affect population dynamics. LTRE's are often summarized by using the rate of increase, $\lambda$, as a demographic statistic to integrate the treatment effects on survival and reproduction throughout the life cycle.

Knowing that a treatment produces a particular value of $\lambda$ leaves unresolved the question of how the manifold changes in the vital rates contribute to the effect on growth rate. After all, some vital rates can be changed a great deal without affecting $\lambda$ (e.g., the survival of a post-reproductive age class), whereas small changes in other vital rates produce large changes in $\lambda$. In addition, most environmental factors have differential effects on the different vital rates. A given treatment may affect survival, growth, and fertility differently, with different effects on those rates in different stages.

Treatment effects on $\lambda$ can be decomposed into contributions from the effects on each of the vital rates (Caswell 1989b, 1996a). This decomposition makes it possible to pinpoint where in the life history the treatment has its greatest impact. The decomposition uses a first-order linear approximation to the effect on $\lambda$. I outline the simplest case here: a set of $M$ treatments $T_{m}$, $m=1, \ldots, M$, each of which produces its own matrix $\mathbf{A}_{m}$ and population growth rate $\lambda^{(m)}$. (I use parenthetical superscripts to denote treatments when subscripts distinguish matrix elements.) 
Choose some condition as a reference treatment; this might be the mean of all the treatments, a control treatment, or any other condition of particular interest. The reference matrix is denoted $\mathbf{A}_{r}$, and treatment effects on $\lambda$ are measured relative to $\lambda^{(r)}$.

To first order, we can write

$$
\lambda^{(m)}-\left.\lambda^{(r)} \approx \sum_{i j}\left(a_{i j}^{(m)}-a_{i j}^{(r)}\right) \frac{\partial \lambda}{\partial a_{i j}}\right|_{\left(\mathbf{A}_{m}+\mathbf{A}_{r}\right) / 2}
$$

for $m=1, \ldots, M$. Each term in the summation gives the contribution of the effect of treatment $m$ on one of the vital rates to its overall effect on $\lambda$. That contribution is the product of the vitalrate effect and the sensitivity of $\lambda$ to changes in that vital rate. If either of these terms is small-if the treatment doesn't effect $a_{i j}$ or if $\lambda$ is insensitive to $a_{i j}$-then the contribution of effects on $a_{i j}$ to effects on $\lambda$ is small. The converse is also true.

The sensitivities in (34) must be calculated from some particular matrix; here they are calculated from a matrix "halfway between" the two matrices ( $\mathbf{A}_{m}$ and $\mathbf{A}_{r}$ ) being compared. There is some theoretical justification for this (Caswell 19896), and it works well in practice.

The reason for using $\lambda$ as a statistic to summarize the results of an LTRE is that it integrates the diverse and stage-specific effects of the treatments. The decomposition analysis complements this use; it pinpoints the source, within the life cycle, of the effects on $\lambda$. Experience with this kind of analysis shows that it is not safe to assume that the biggest changes in vital rates are responsible for the effects of a treatment on $\lambda$. Without some analysis like (34), half of the information contained in an LTRE is wasted.

Equation (34) describes a simple, one-way, fixed-effect experimental design. The approach has been extended to factorial designs, random designs, and regression designs (Caswell, in press). It can also be applied to statistics other than $\lambda$ (as long as a perturbation theory is available for the statistic) and to parameters other than matrix elements (Caswell 1989c, 1996a, in press).

\section{Prospective and Retrospective Analyses}

The preceding subsections outline two ways of using perturbation analysis. Sensitivity and elasticity calculations are prospective analyses; they predict the results of perturbations of the vital rates before they happen. Indeed, they even show the results of perturbations that are biologically impossible. They tell nothing about 
which vital rates are actually responsible for an observed change in $\lambda$. The LTRE decomposition analysis answers this kind of retrospective question. It does so by combining sensitivity analysis with information on the actual variance in the $a_{i j}$.

Don't confuse these two kinds of analysis, especially in ambiguous questions such as, "which of the vital rates is most important to population growth?" One way to answer this question is to find the rate with the biggest sensitivity (or elasticity); that rate is most important in the sense that if you were to change all the rates by the same amount (or same proportion), it would have the biggest impact. Another answer is based on an LTRE analysis; the most important vital rate is the one with the variation that makes the biggest contribution to the variability in $\lambda$. The two answers are usually different. Both are valid, but they answer different questions, the first prospective, the second retrospective. Chapter 7 , by Horvitz et al., explores these issues further.

\section{Density-Dependent Matrix Models}

The models analyzed so far have been linear and time-invariant; the vital rates are independent of population density or temporal changes. Time-varying models are discussed in Chapter 3 ; here I consider the inclusion of density dependence, which makes the model nonlinear. Although the mathematical tools are different from those used in the linear case, the focus is still on asymptotic behavior and perturbation. Unlike linear models, densitydependent models do not grow exponentially. Instead, solutions tend to converge to limited subsets of the state space, called attractors. The attractors may be fixed points (also called equilibria), cycles, or more-complicated structures.

Two kinds of perturbation analysis are important. One asks the effect of perturbing initial conditions. This is of no interest in the linear case, since the ergodic theorem guarantees convergence to the stable population structure and asymptotic growth rate from any nonzero initial condition. In a density-dependent model, however, small perturbations of initial conditions can lead to very different dynamics, depending on the stability of a fixed point. The second kind of perturbation considers changes in the parameters of the model. Often, small changes in parameters leave the qualitative asymptotic behavior unchanged. But sometimes small parameter changes have big effects on asymptotic dynamics: stable trajectories become unstable, fixed points are created or destroyed, attrac- 
tors change from fixed points to cycles, cycles give way to chaos, etc. These qualitative changes are called bifurcations, and finding them is a major pastime of people who study density-dependent models.

There are many ways of incorporating density dependence in matrix models (Caswell 1989a; see also Cushing 1988; Getz \& Haight 1989; Silva \& Hallam 1992; Logofet 1993; Dennis et al. 1995). "Density" can be defined as the abundance of a stage or set of stages, as a weighted combination of the abundances of a set of stages, or simply as the total number $N=\sum n_{i}$. Density can affect the vital rates at many points in the life cycle or at only one. Each of the vital rates may be a different function of the density, or the entire set of vital rates may be affected by density in the same way. In this section, I introduce three models that incorporate simple density effects on reproduction, on growth, and on survival, using them to demonstrate some dynamic consequences of density dependence.

\section{Basic Formulations}

Consider a two-stage model, with a matrix

$$
\mathbf{A}=\left[\begin{array}{ll}
P_{1} & F_{2} \\
G_{1} & P_{2}
\end{array}\right]
$$

and suppose that the matrix entries are given by

$$
\begin{aligned}
& P_{1}=\sigma_{1}\left(1-\gamma_{1}\right) . \\
& G_{1}=\sigma_{1} \gamma_{1} . \\
& P_{2}=\sigma_{2} .
\end{aligned}
$$

where $\sigma_{1}$ and $\sigma_{2}$ are survival probabilities, and $i_{1}$ is the protuatility of growth from stage 1 to stage 2 , approximated by $1 / \tau_{1}$. where $\tau_{1}$ is the mean duration of stage 1 .

This simple model contains the rates of reproduction, growth, and survival, each of which can be made density-dependent.

Density-dependent reproduction. Let fertility at zero density be given by $f_{0}$, and suppose that fertility declines exponentially with increases in density. Then,

$$
F_{2}(N)=f_{0} \exp (-b N),
$$


where $b$ is a constant that measures the strength of density dependence. The resulting matrix is

$$
\mathbf{A}^{(\mathrm{f})}=\left[\begin{array}{cc}
P_{1} & f_{0} e^{-6 N} \\
G_{1} & P_{2}
\end{array}\right] .
$$

Density-dependent survival. Let $\sigma_{1,0}$ and $\sigma_{2,0}$ denote the survival probabilities of stages 1 and 2 at zero density. Assume that both juvenile and adult survival probabilities are affected in the same way by density, so that

$$
\begin{aligned}
& \sigma_{1}(N)=\sigma_{1,0} \exp (-b N) \\
& \sigma_{2}(N)=\sigma_{2,0} \exp (-b N) .
\end{aligned}
$$

The resulting matrix is

$$
\begin{aligned}
\mathbf{A}^{(\mathrm{s})} & =\left[\begin{array}{cc}
\sigma_{1,0}\left(1-\gamma_{1}\right) e^{-b N} & F_{2} \\
\sigma_{1,0} \gamma_{1} e^{-b N} & \sigma_{2,0} e^{-b N}
\end{array}\right] \\
& =\left[\begin{array}{cc}
P_{1,0} e^{-b_{1} N} & F_{2} \\
G_{1,0} e^{-b_{1} N} & P_{2,0} e^{-b_{2} N}
\end{array}\right] .
\end{aligned}
$$

Density-dependent growth. Suppose that the mean duration of the juvenile stage increases with density:

$$
\tau(N)=\tau_{0} \exp (b N)
$$

then the growth probability is given by

$$
\begin{aligned}
\gamma(N) & =\frac{1}{\tau(N)} \\
& =\gamma_{0} \exp (-b N) .
\end{aligned}
$$

The resulting matrix is

$$
\begin{aligned}
\mathbf{A}^{(\mathrm{g})} & =\left[\begin{array}{cc}
\sigma_{1}\left(1-\gamma_{0} e^{-b N}\right) & F_{2} \\
\sigma_{1} \gamma_{0} e^{-b N} & P_{2}
\end{array}\right] \\
& =\left[\begin{array}{cc}
\sigma_{1}\left(1-\gamma_{0} e^{-b N}\right. & F_{2} \\
G_{1,0} e^{-b N} & P_{2}
\end{array}\right] .
\end{aligned}
$$

The assumption of exponential density dependence ("overcompensation" in the language of fisheries biology) has important dynamic consequences. A strictly compensatory form (e.g., $1 /(1+b N)$ ), or a depensatory form with some range of positive density dependence, may produce different dynamics (Caswell 1989a; Silva \& Hallam 1992). 


\section{Equilibria and Stability}

The equilibria of a density-dependent model are population vectors $\hat{\mathbf{n}}$ that satisfy

$$
\hat{\mathbf{n}}=\mathbf{A}_{\hat{n}} \hat{\mathbf{n}} .
$$

Depending on the form of the density dependence, there may be more than one equilibrium. In the absence of immigration, $\hat{\mathbf{n}}=$ 0 is always an equilibrium. With a little luck, equation (43) can be solved analytically for $\hat{\mathbf{n}}$. More often than not, however, the equilibria must be found numerically.

Density-dependent reproduction. The equilibrium $\hat{\mathbf{n}}$ is defined by

$$
\begin{aligned}
& \hat{n}_{1}=P_{1} \hat{n}_{1}+f_{0} e^{-b \hat{N}_{n}} \hat{n}_{2}, \\
& \hat{n}_{2}=G_{1} \hat{n}_{1}+P_{2} \hat{n}_{2} .
\end{aligned}
$$

The second of these equations can be solved for $\hat{n}_{1}$ :

$$
\hat{n}_{1}=\frac{1-P_{2}}{G_{1}} \hat{n}_{2}
$$

substituting this in the first equation yields

$$
\hat{N}=-\frac{1}{b} \log \left(\frac{\left(1-P_{1}\right)\left(1-P_{2}\right)}{f_{0} G_{1}}\right) .
$$

Combining these two results gives an expression for $\hat{n}_{2}$,

$$
\hat{n}_{2}=\frac{G_{1} \hat{N}}{\left(1+G_{1}-P_{2}\right)} ;
$$

and $\hat{n}_{1}$ can be found as $\hat{n}_{1}=\hat{N}-\hat{n}_{2}$.

Density-dependent survival. The equations defining the equilibrium are

$$
\begin{aligned}
& \hat{n}_{1}=P_{1,0} e^{-b \hat{N}} \hat{n}_{1}+F_{2} \hat{n}_{2}, \\
& \hat{n}_{2}=G_{1,0} e^{-b \hat{N}} \hat{n}_{1}+P_{2,0} e^{-b \hat{N}} \hat{n}_{2} .
\end{aligned}
$$

The second of these equations can be solved for $\hat{n}_{2}$ :

$$
\hat{n}_{2}=\frac{G_{1,0} e^{-b \hat{N}}}{\left(1-P_{2,0}\right) e^{-b \hat{N}}} \hat{n}_{1} .
$$

When substituted into the first equation this eventually yields

$$
P_{1,0}-P_{1.0} P_{2,0} e^{-b \hat{N}}+F_{2} G_{1,0}+P_{2,0}=e^{b \hat{N}} \text {. }
$$


Multiplying both sides by $e^{b \hat{N}}$ gives a quadratic equation in the new variable $y=e^{b \hat{N}}$. Solving this equation for $\hat{y}$ and substituting leads to

$$
\begin{aligned}
& \hat{N}=\log \hat{y} b, \\
& \hat{n}_{1}=\log \hat{y}\left(b\left(1+\frac{G_{1,0}}{\hat{y}-P_{2,0}}\right)\right)^{-1}, \\
& \hat{n}_{2}=\hat{N}-\hat{n}_{1} .
\end{aligned}
$$

Density-dependent growth. The equations to be solved for the equilibrium are

$$
\begin{aligned}
& \hat{n}_{1}=\sigma_{1}\left(1-\gamma_{0} e^{-b \hat{N}}\right) \hat{n}_{1}+F_{2} \hat{n}_{2}, \\
& \hat{n}_{2}=\sigma_{1} \gamma_{0} e^{-b \hat{N}} \hat{n}_{1}+P_{2} \hat{n}_{2} .
\end{aligned}
$$

As before, the second equation can be solved for $\hat{n}_{2}$,

$$
\hat{n}_{2}=\frac{\sigma_{1} \gamma_{0} e^{-b \hat{N}}}{1-P_{2}} \hat{n}_{1}
$$

substituting this relation into the first equation eventually leads to

$$
\hat{N}=-\frac{1}{b} \log \left(\frac{\left(1-\sigma_{1}\right)\left(1-P_{2}\right)}{\sigma_{1} \gamma_{0}\left(F_{2}+P_{2}-1\right)}\right)
$$

None of these analytical solutions for $\hat{\mathbf{n}}$ is particularly informative at first glance. This is typical; only in exceptional circumstances are the formulas for the equilibria in a matrix population model simple enough to appear informative.

An equilibrium is said to be locally stable if small perturbations remain close to the equilibrium, and locally asymptotically stable if small perturbations eventually return to the equilibrium. The adjective "local" refers to the smallness of the perturbations; it may well happen that small perturbations return to an equilibrium, but large ones are attracted to another equilibrium, or to some other kind of attractor (a cycle, for example, or a strange attractor).

The local stability of a fixed point is determined by approximating the nonlinear density-dependent model by a linear model that is accurate for small perturbations. Begin by defining a vector $\mathbf{x}$ of deviations from the equilibrium $\hat{\mathbf{n}}$ :

$$
\mathbf{x}(t)=\mathbf{n}(t)-\hat{\mathbf{n}} .
$$


The dynamics of $\mathbf{x}$, near the equilibrium, are given by

$$
\mathbf{x}(t+1)=\mathbf{B} \mathbf{x}(t) .
$$

where the constant matrix $\mathbf{B}$, called the Jacobian matrix, is the linear approximation to the full nonlinear system near the equilibrium.

To illustrate, let us write a two-dimensional matrix model as

$$
\begin{aligned}
& n_{1}(t+1)=a_{11} n_{1}(t)+a_{12} n_{2}(t)=f_{1}(\mathbf{n}), \\
& n_{2}(t+1)=a_{21} n_{1}(t)+a_{22} n_{2}(t)=f_{2}(\mathbf{n}),
\end{aligned}
$$

with the understanding that $a_{i j}=a_{i j}(\mathbf{n})$.

The Jacobian matrix $\mathbf{B}$ is

$$
\mathbf{B}=\left[\begin{array}{ll}
\frac{\partial f_{1}}{\partial n_{1}} & \frac{\partial f_{1}}{\partial n_{2}} \\
\frac{\partial f_{2}}{\partial n_{1}} & \frac{\partial f_{2}}{\partial n_{2}}
\end{array}\right] .
$$

Differentiating $f_{1}$ gives

$$
\begin{aligned}
& \frac{\partial f_{1}}{\partial n_{1}}=a_{11}+n_{1} \frac{\partial a_{11}}{\partial n_{1}}+n_{2} \frac{\partial a_{12}}{\partial n_{1}}, \\
& \frac{\partial f_{1}}{\partial n_{2}}=a_{12}+n_{1} \frac{\partial a_{11}}{\partial n_{2}}+n_{2} \frac{\partial a_{12}}{\partial n_{2}} .
\end{aligned}
$$

The derivatives of $f_{2}$ have the same form. Thus, $\mathbf{B}$ is given by

$$
\begin{aligned}
& \mathbf{B}=\mathbf{A}_{\hat{n}}+\left[\begin{array}{ll}
n_{1} \frac{\partial a_{11}}{\partial n_{1}}+n_{2} \frac{\partial a_{12}}{\partial n_{1}} & n_{1} \frac{\partial a_{11}}{\partial n_{2}}+n_{2} \frac{\partial a_{12}}{\partial n_{2}} \\
n_{1} \frac{\partial a_{21}}{\partial n_{1}}+n_{2} \frac{\partial a_{22}}{\partial n_{1}} & n_{1} \frac{\partial a_{21}}{\partial n_{2}}+n_{2} \frac{\partial a_{22}}{\partial n_{2}}
\end{array}\right] \\
& =\mathbf{A}_{\hat{n}}+\left[\begin{array}{ll}
\frac{\partial \mathbf{A}}{\partial n_{1}} \hat{\mathbf{n}} & \frac{\partial \mathbf{A}}{\partial n_{2}} \hat{\mathbf{n}}
\end{array}\right] .
\end{aligned}
$$

All of the derivatives are evaluated at the equilibrium.

Equation (63) makes the calculation of the Jacobian straightforward for matrix population models (it is due to Beddington 1974). Given the equilibrium vector $\hat{\mathbf{n}}$, calculate the derivatives of $\mathbf{A}$ with respect to each of the $n_{i}$ and multiply these matrices by $\hat{\mathbf{n}}$; put the resulting vectors as columns in a matrix, and add this to $\mathbf{A}$ evaluated at the equilibrium.

The equilibrium $\hat{\mathbf{n}}$ is asymptotically stable if the eigenvalues of $\mathbf{B}$ are all less than one in magnitude (or are "within the unit circle," referring to the circle with radius one in the complex plane). It is unstable if any of the eigenvalues are outside the unit circle. An eigenvalue falling exactly on the unit circle (i.e., with magnitude exactly equal to one) signals a transition from stability to 
instability, or vice versa, and is considered in the next section.

Remember that (63) describes the dynamics of perturbations, not the dynamics of numbers of individuals. Thus, the entries of $\mathbf{x}$ can be negative, and the matrix $\mathbf{B}$ often contains negative entries. The Perron-Frobenius theorem is of little use in evaluating the eigenvalues of B, except in special cases (DeAngelis et al. 1980; Caswell 1989a, Example 9.3), and the eigenvalue of largest magnitude may be negative or complex.

The Jacobian matrices for our three density-dependent models can be written down easily. Note that all density effects depend on $N=n_{1}+n_{2}$, so that

$$
\frac{\partial a_{i j}}{\partial n_{1}}=\frac{\partial a_{i j}}{\partial n_{2}}=\frac{\partial a_{i j}}{\partial N}
$$

Thus, the matrix $\mathbf{B}$ reduces to

$$
\mathbf{B}=\mathbf{A}_{\hat{n}}+\frac{\partial \mathbf{A}}{\partial N}\left[\begin{array}{ll}
\hat{n}_{1} & \hat{n}_{1} \\
\hat{n}_{2} & \hat{n}_{2}
\end{array}\right]
$$

The resulting Jacobian matrices for the three example models are as follows: for density-dependent reproduction,

$$
\mathbf{B}=\left[\begin{array}{cc}
P_{1} & f_{0} e^{-b \hat{N}} \\
G_{1} & P_{2}
\end{array}\right]-b f_{0} e^{-b \hat{N}}\left[\begin{array}{cc}
\hat{n}_{2} & \hat{n}_{2} \\
0 & 0
\end{array}\right]
$$

for density-dependent survival,

$$
\begin{aligned}
\mathbf{B}= & {\left[\begin{array}{cc}
P_{1,0} e^{-b \hat{N}} & F_{2} \\
G_{1,0} e^{-b \hat{N}} & P_{2,0} e^{-b \hat{N}}
\end{array}\right] } \\
& -b e^{-b \hat{N}}\left[\begin{array}{cc}
P_{1,0} \hat{n}_{1} & P_{1,0} \hat{n}_{1} \\
G_{1,0} \hat{n}_{1}+P_{2,0} \hat{n}_{2} & G_{1,0} \hat{n}_{1}+P_{2,0} \hat{n}_{2}
\end{array}\right] ;
\end{aligned}
$$

and for density-dependent growth,

$$
\begin{aligned}
\mathbf{B}= & {\left[\begin{array}{cc}
\sigma_{1}\left(1-\gamma_{0} e^{-b \hat{N}}\right) & F_{2} \\
G_{1,0} e^{-b \hat{N}} & P_{2}
\end{array}\right] } \\
& +b G_{1,0} e^{-b \hat{N}}\left[\begin{array}{cc}
\hat{n}_{1} & \hat{n}_{1} \\
-\hat{n}_{1} & -\hat{n}_{1}
\end{array}\right] .
\end{aligned}
$$

In each case, $\hat{N}$ and $\hat{\mathbf{n}}$ are evaluated using the appropriate equilibrium formulas. Note that I have not attempted to insert the formulas for the equilibria. In general, it is a lucky circumstance in which such expressions simplify usefully. More often, the best that can be done is to write the Jacobian in terms of the equilibria and to carry out further analyses numerically. 
In studying a linear model, the response of the eigenvalues to changes in parameters is usually more interesting than their values for one specific parameter set. Similarly, in studying a nonlinear model it is usually more interesting to see how the stability properties change as parameters are varied than to characterize stability for one parameter set. A qualitative change in stability is called a bifurcation.

\section{Bifurcations}

Roger Tory Peterson published the first edition of $A$ Field Guide to the Birds in 1934. At the time, the standard guide for the would-be birdwatcher was Chapman's (1932) Handbook of Birds of Eastern North America, first published in 1895. Chapman's book contained detailed dichotomous keys to the bird species, most of which would be useless without having the bird in one hand (and, most likely, a smoking shotgun in the other). Peterson's innovation was to sacrifice rigor but to focus on characters that would distinguish most of the species, most of the time, in the field.

My goal in this section is to provide a kind of field guide to bifurcations of equilibria of nonlinear matrix models. I describe the basic ideas of bifurcation theory, without assuming that my reader is equipped with the powerful tools of rigorous bifurcation theory, but assuming the ability to conduct numerical simulations. The question is, what to look for that will help to identify the bifurcations that occur.

$\mathrm{Be}$ forewarned that, just as there are situations where Peterson had to admit that a reliance on field marks was misleading or impossible, there are no doubt situations in which my suggestions here will be incorrect. (In a notorious case, Chapman distinguished the Alder and Least flycatchers of the genus Empidonax on the basis of whether the wing is longer or shorter than 2.60 inches and whether the lower mandible is flesh-colored or strongly tinged with brownish. Peterson, by contrast, said that "it is quite risky to attempt to tell them apart by mere variations in color" and relied on calls during the breeding season.) In such cases, you can make use of the many detailed treatments of bifurcation theory (two excellent recent examples are Wiggins 1990 and Hale \& Koçak 1991; see also the review paper Whitley 1983) that provide the mathamatical analogue of the birdwatcher's shotgun. See Chapter 6 , by Cushing, for an introduction to the application of this theory to matrix models. 
Types of bifurcations. An equilibrium point can bifurcate when it loses stability in response to a change in a parameter, that is, when the dominant eigenvalue $\lambda$ of the Jacobian matrix $\mathbf{B}$ in equation (63) is equal to one. This can happen in three ways:

$$
\begin{array}{ll}
\lambda=1, & \text { a " }+1 \text { bifurcation," } \\
\lambda=-1, & \text { a "-1 bifurcation," } \\
\lambda=a \pm b i, & \text { a "complex conjugate pair" bifurcation. }
\end{array}
$$

When an eigenvalue (or a complex conjugate pair) crosses the unit circle, the others must still be inside, or there would be no change in stability, since the equilibrium would already be unstable. (I am ignoring the rare cases in which more than one eigenvalue crosses the unit circle simultaneously.) Perhaps surprisingly, the bifurcation does not depend on what those other eigenvalues are doing, nor on how many of them there are. The mathematical expression of this fact is the Center Manifold Theorem (e.g., Wiggins 1990). Roughly, it says that the state space can be divided into two parts, one associated with the eigenvalue leaving the unit circle and the second associated with all the other eigenvalues. The dynamics on the second part remain stable, while the bifurcation can be studied on the first part. Thus all the basic behaviors of +1 and -1 bifurcations can be studied in one-dimensional models, while the Hopf bifurcation can be studied in two-dimensional models.

What different bifurcations are possible in response to a parameter change? First, +1 bifurcations are of three types.

1. At a transcritical bifurcation, two fixed points, one stable and the other unstable, collide and exchange stability. This occurs in all density-dependent matrix models as a bifurcation of the zero equilibrium. On one side of the bifurcation the zero equilibrium is stable. An unstable equilibrium exists, but it is negative and usually ignored in our thinking about populations. However, it does exist. On the other side of the bifurcation, the zero equilibrium is unstable, and there is a stable positive equilibrium. See Chapter 6, by Cushing, for a detailed description of this bifurcation.

2. On one side of the saddle-node bifurcation, there is no fixed point at all. At the bifurcation point, two fixed points appear, one stable and the other unstable. What you see in a saddlenode bifurcation depends on where you look. If you follow the stable fixed point, it suddenly disappears (as it collides with the 
unstable fixed point of which you were blissfully unaware), and trajectories move off to some other attractor in the state space.

Saddle-node bifurcations occur in population models when there is an Allee effect (also known as depensatory density dependence) present, that is, when, at low densities, survival or fertility depends positively on density. Typically, such a population has three fixed points: zero, an intermediate critical density, and a high density. Zero and the high density are both stable fixed points, while the intermediate critical density is unstable. Populations below the critical density decay to extinction, and those above it increase to the high-density fixed point. As some parameter is varied, the high-density fixed point and the intermediatedensity fixed point collide and annihilate each other; the population at that point crashes to extinction. Cushing (Chapter 6) shows an example.

3. On one side of a pitchfork bifurcation, there is one stable fixed point. On the other side, one unstable fixed point is surrounded by two new, stable fixed points. At the bifurcation, the two stable fixed points appear close to the now-unstable fixed point; a trajectory may approach either of the two stable fixed points, depending on initial conditions. The canonical examples of pitchfork bifurcations occur in one-dimensional cubic maps. These maps appear in some genetic models (May 1979), but pitchfork bifurcations rarely appear in density-dependent matrix models.

Second are -1 bifurcations, or fip bifurcations. At this type of bifurcation point, a stable fixed point becomes unstable, and a stable 2-cycle appears. Just beyond the bifurcation point, the amplitude of the 2-cycle is small, and it surrounds the unstable fixed point.

The third type of bifurcation develops from complex-conjugate pairs of eigenvalues and is also called a Hopf or Naimark-Sacker bifurcation. As a complex conjugate pair of eigenvalues leave the unit circle, the fixed point loses its stability and an invariant circle forms around the now-unstable fixed point. An invariant circle is a continuous closed curve (not necessarily an exact circle). Any point on this curve is mapped to another point on the curve, hence the term "invariant."

Trajectories on the invariant circle may be periodic, or they may be quasiperiodic, rotating around the circle without ever repeating themselves, depending on the location of the eigenvalues when they cross the unit circle at the bifurcation. In general, trajecto- 
ries immediately following a Hopf bifurcation are quasiperiodic, containing two periods that are not integer multiples of each other (see example below).

There are two other possibilities. One is called strong resonance and occurs when the bifurcation point happens at

$$
\lambda=e^{2 \pi k i}, \quad \text { where } \quad k=1,1 / 2,1 / 3,1 / 4 .
$$

The second is called weak resonance and occurs when

$$
\lambda=e^{2 \pi i p / q}
$$

where $p / q$ is a ratio of small integers but does not equal any of the first four roots of unity. In this case, the trajectory on the invariant circle has period $q$.

Strong resonance is complicated. Mathematicians say things like "the strong resonances ... exhibit rather different behavior, the details of which are not yet fully understood" (Whitley 1983), or "the dynamics of such maps ... can be exceedingly complicated and the answer is not yet completely known" (Hale \& Koçak 1991). I cannot improve on that, but it appears that bifurcations with strong resonance often have trajectories with period $1 / k$ lurking in their dynamics, often mixed up with quasiperiodic and, eventually, chaotic dynamics (for an example, see Guckenheimer et al. 1977).

Examples: bifurcations of the zero equilibrium. The three simple density-dependent models in this section provide examples of these bifurcations. Zero is an equilibrium of all three models; to examine its bifurcations, evaluate the Jacobian matrix at zero and track the magnitude of its eigenvalues as parameters in the matrix change. In the examples below, I show bifurcations resulting from changes in reproductive output $F_{2}$ (or $f_{0}$ in the model with density-dependent fertility). Note, however, that Cushing (1988; Chapter 6) has shown in general that the bifurcation parameter can be taken to be the net reproductive rate (the expected number of offspring produced by an individual over its lifetime); he has shown how to calculate this quantity for arbitrary stage-structured matrices.

The Jacobian matrix for the zero equilibrium is particularly simple, because the second term on the right-hand side of equation (63) is zero. Thus, the Jacobian is simply the projection matrix evaluated at zero, and it is the same in all three examples.

Figure 3 shows the magnitude of the dominant eigenvalue of $\mathbf{B}$ as a function of $F_{2}$; it exceeds one when $F_{2} \approx 0.8$. The lower panel shows the location in the complex plane of the dominant 

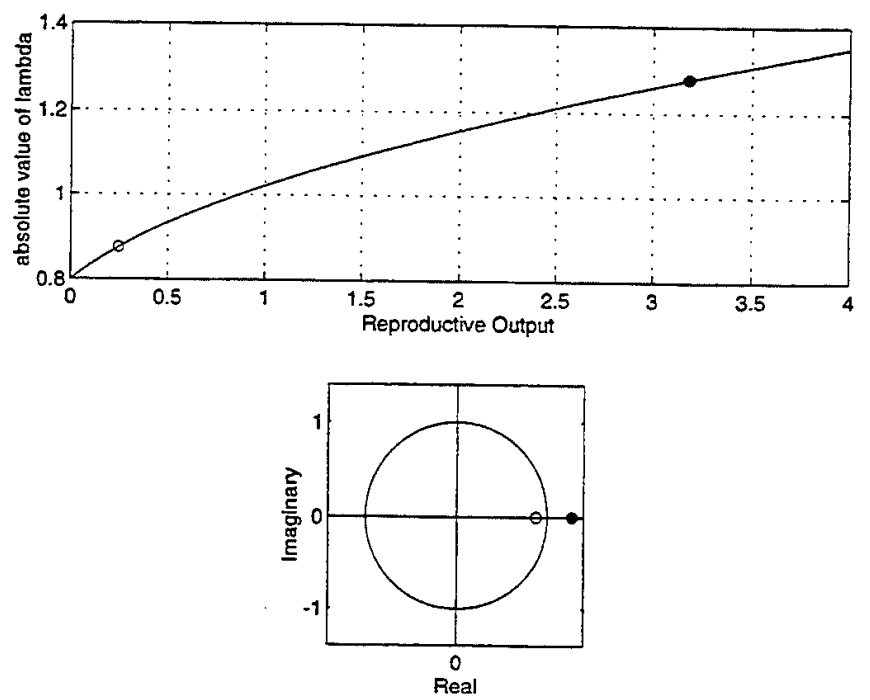

FIGURE 3. The bifurcation of the zero equilibrium for the three twostage examples. Upper panel, The magnitude of the dominant eigenvalue of the Jacobian matrix $\mathbf{B}$ as a function of the reproductive output $F_{2}$. Lower panel. The location, relative to the unit circle in the complex plane, of the eigenvalue just before (open circle) and after (closed circle) the bifurcation. Parameter values: $\sigma_{1}=0.6, \sigma_{2}=0.8, \gamma_{1,0}=0.2, b=1$.

eigenvalue before and after the bifurcation; this is a +1 bifurcation since the eigenvalue leaves the unit circle at +1 . Figure 4 shows the equilibrium (for total population size $N=n_{1}+n_{2}$ ) as a function of $F_{2}$. The pattern is typical of a transcritical bifurcation. Note that all three models bifurcate from zero at the same value of $F_{2}$, because all three matrices are identical evaluated at $\hat{\mathbf{n}}=0$, even though they differ in their subsequent behavior.

Bifurcations of the positive equilibrium. What happens to the stable positive equilibrium that appears after the zero equilibrium becomes unstable? Each of the three models shows a different bifurcation pattern. I show examples for each model, using $F_{2}$ (or $f_{0}$ in the model with density-dependent fertility) as the bifurcation parameter. There is no reason to expect that this exhausts the possibilities; other types of bifurcations may well be produced by varying other parameters.

For density-dependent reproduction, the bifurcation occurs when $f_{0} \approx 95$. The eigenvalue leaves the unit circle at -1 (Fig. 5), 

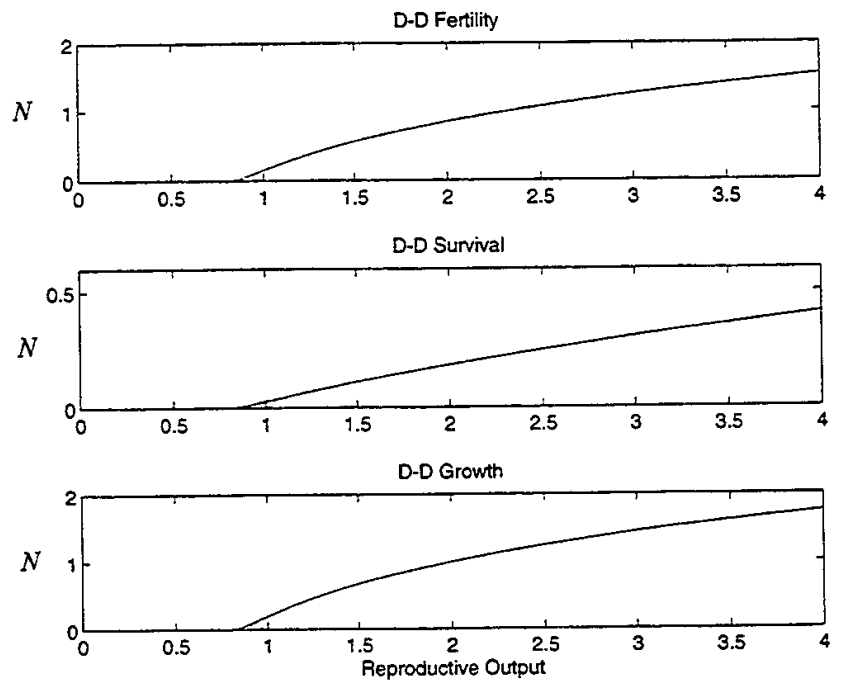

FIGURE 4. Bifurcation plots for the three two-stage examples, showing the equilibrium value of $N=n_{1}+n_{2}$ as a function of reproductive output $\left(F_{2} ;\right.$ in the model with density-dependent fertility, $\left.f_{0}\right)$. Other parameter values as in Figure 3.
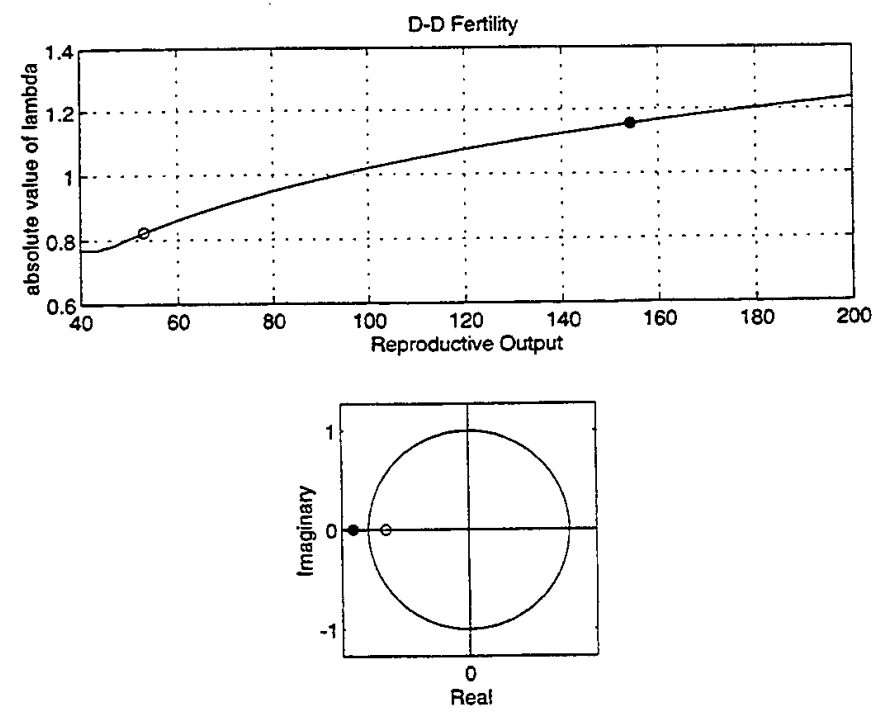

FIGURE 5. The bifurcation of the positive equilibrium for the densitydependent fertility model as a function of reproductive output $f_{0}$. Interpretation and parameter values as in Figure 3. 

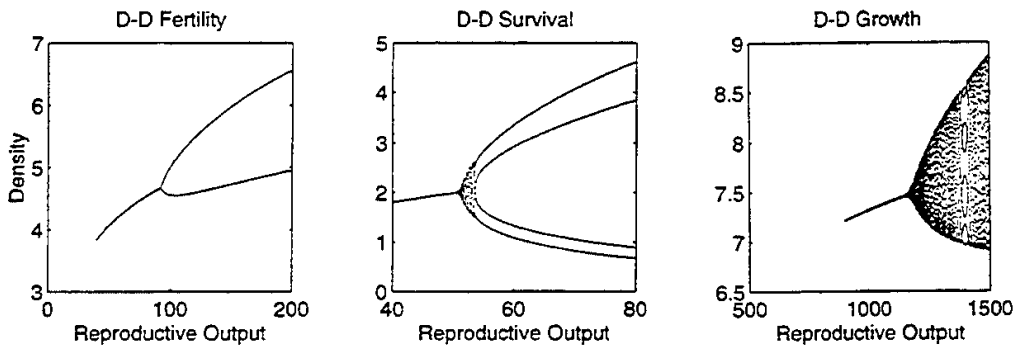

FIGURE 6. Bifurcation plots for the three two-stage examples, showing the long-term behavior of total population size $\left(N=n_{1}+n_{2}\right)$ as a function of reproductive output ( $F_{2}$; for the model with density-dependent fertility, $\left.f_{0}\right)$. Other parameters as in Figure 3.

implying a flip bifurcation, as seen in Figure 6a. The fixed point is replaced by a 2-cycle.

For the model with density-dependent survival, the equilibrium becomes unstable when $F_{2} \approx 50$. The eigenvalue leaves the unit circle very near (but not quite on) the imaginary axis (Fig. 7). This implies a Hopf bifurcation, but one very close to strong resonance. The bifurcation pattern is shown in Figure $6 b$. Just after the bifurcation point, trajectories are quasiperiodic on an invariant circle. As $F_{2}$ increases further, the invariant circle begins to look more like an invariant square, finally collapsing to a stable 4-cycle (Fig. 8). See Wikan and Mjolhus (1995) for an exploration of this period4 behavior in a fully age-classified model with density-dependent survival.

For the model with density-dependent growth, the bifurcation occurs when $F_{2} \approx 1200$. This time, the eigenvalues leave the unit circle as a complex conjugate pair (Fig. 9) that is not one of the first four roots of unity. The result is a Hopf bifurcation to quasiperiodic dynamics on an invariant circle (Fig. 10).

Subcritical and supercritical bifurcations. The flip, pitchfork, and Hopf bifurcations come in two varieties, the supercritical (which I have discussed so far) and the subcritical. For purposes of this discussion, suppose that the bifurcation happens when $f=f_{c}$, and that the fixed point is stable for $f<f_{\mathrm{c}}$ and unstable for $f>f_{\mathrm{c}}$. In the supercritical bifurcations, the unstable fixed point for $f>f_{\mathrm{c}}$ is accompanied by a stable 2-cycle, two stable fixed points, or a stable invariant circle. In the subcritical bifurcations, the 2-cycle, 

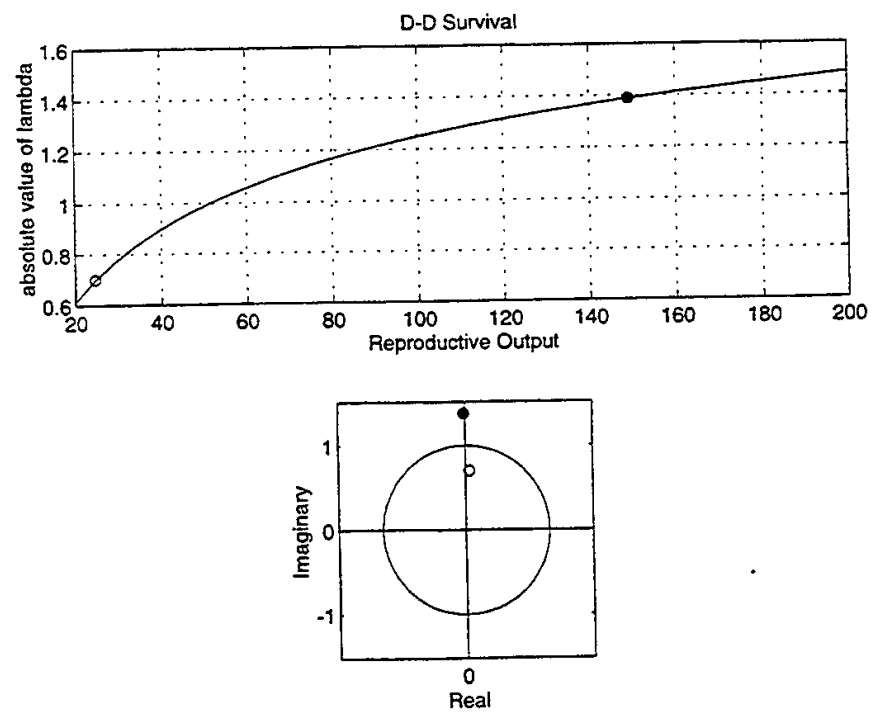

FIGURE 7. The bifurcation of the positive equilibrium for the model with density-dependent survival as a function of reproductive output $F_{2}$. Interpretation and parameter values as in Figure 3.

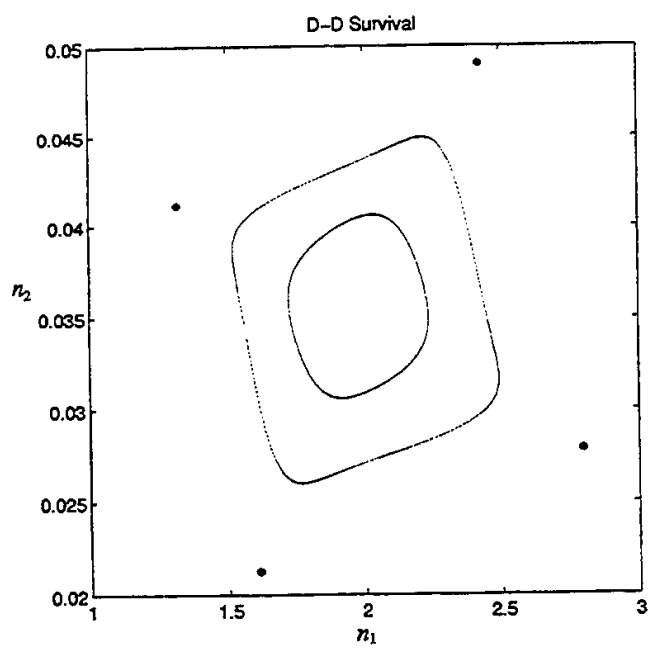

FIGURE 8. Phase portraits for the asymptotic dynamics of the model with density-dependent survival. The inner invariant curve is for $F_{2}=52$; the outer curve, $F_{2}=53$; the 4 -cycle, $F_{2}=55$. Other parameters as in Figure 3. 

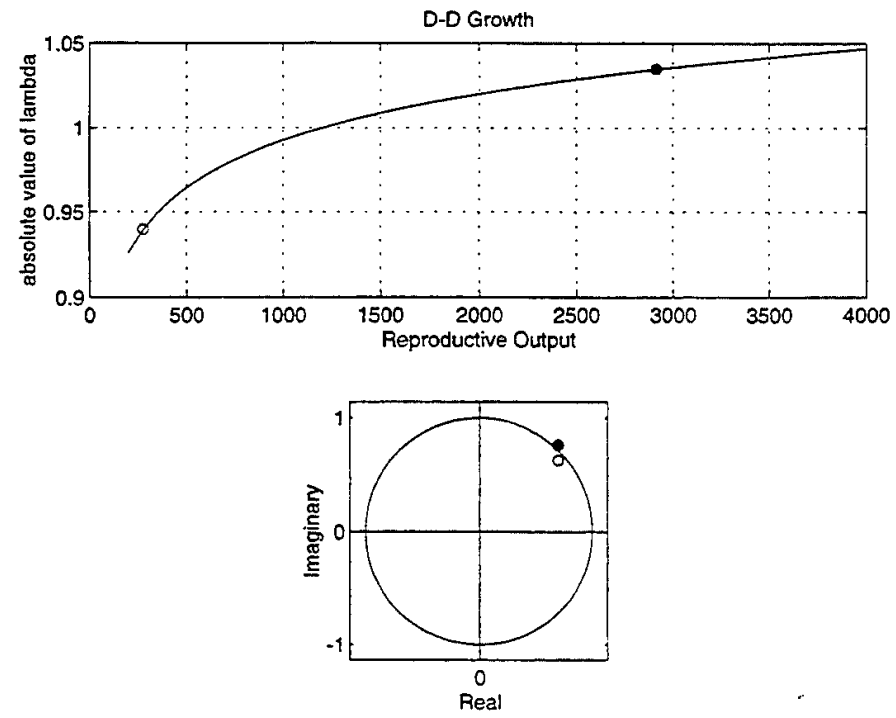

FIGURE 9. The bifurcation of the positive equilibrium for the model with density-dependent growth as a function of reproductive output $F_{2}$. Interpretation and parameter values as in Figure 3.

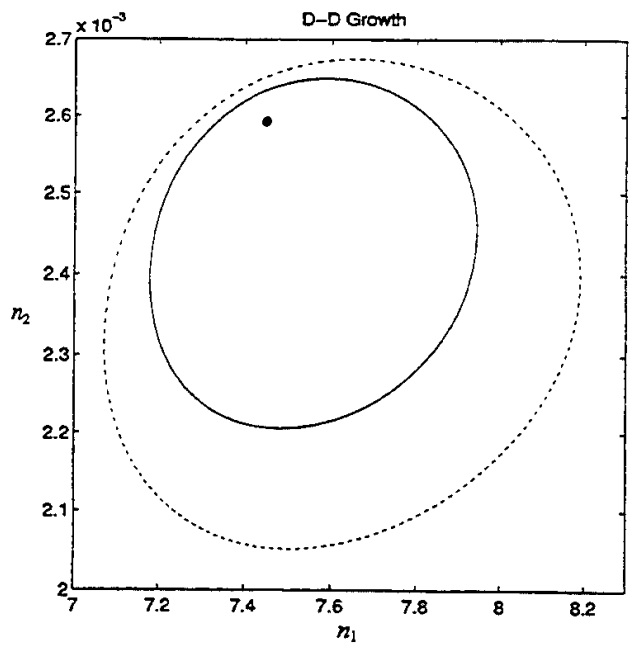

FIGURE 10. Phase portraits for the asymptotic dynamics of the model with density-dependent growth. The inner equilibrium point is for $F_{2}=$ 1150 ; the invariant curves are for $F_{2}=1250$ and $F_{2}=1300$. Other parameters as in Figure 3. 
the two fixed points, or the invariant circle exist for $f<f_{c}$, but they are unstable. At the bifurcation point $f_{\mathrm{c}}$, the original fixed point becomes unstable and the other structures disappear.

What happens after a subcritical bifurcation (i.e., for $f>f_{c}$ ) is hard to predict. Since the fixed point becomes unstable at $f_{\mathrm{c}}$, trajectories do not remain close by, but no nearby stable fixed points, cycles, or invariant circles appear to attract trajectories. Therefore, solutions leave the vicinity of the original fixed point, and their dynamics depend on the location of other attractors in the system.

Subcritical bifurcations are not as common as supercritical ones, but they cannot be ignored. Neubert and Kot (1992) found that, in four discrete predator-prey models in which the prey alone exhibited a supercritical fip bifurcation, the system with both predator and prey exhibited a subcritical flip bifurcation, leading to extinction of the predator. Guckenheimer et al. (1977) and Levin (1981) found subcritical bifurcations in age-structured models with strongly resonant Hopf bifurcations, and Dennis et al. (1995) found a subcritical bifurcation in a stage-structured model fit to laboratory data on Tribolium populations.

Analytical methods exist for determining whether a bifurcation is supercritical or subcritical, on the basis of terms in the Taylor series expansion of the model near the fixed point. However, these methods require that the model first be reduced to one or two dimensions using the Center Manifold Theorem, which is not always easy.

On beyond the first bifurcation. The positive fixed point of a matrix population model usually first loses stability through a supercritical flip or supercritical Hopf bifurcation and, then, is replaced by a 2-cycle or a quasiperiodic trajectory on an invariant circle. As the bifurcation parameter increases further, these attractors go through a series of bifurcations, often leading to chaos. The fip bifurcation is typically followed by a series of period-doublings. Successively higher periods are stable for smaller ranges of the bifurcation parameter; eventually, trajectories become chaotic. The resulting bifurcation diagram is similar to the familiar diagram for the discrete logistic equation.

Quasiperiodic solutions produced by a Hopf bifurcation usually go through a series of frequency locking events in which trajectories become periodic for a range of values of the bifurcation parameter. 

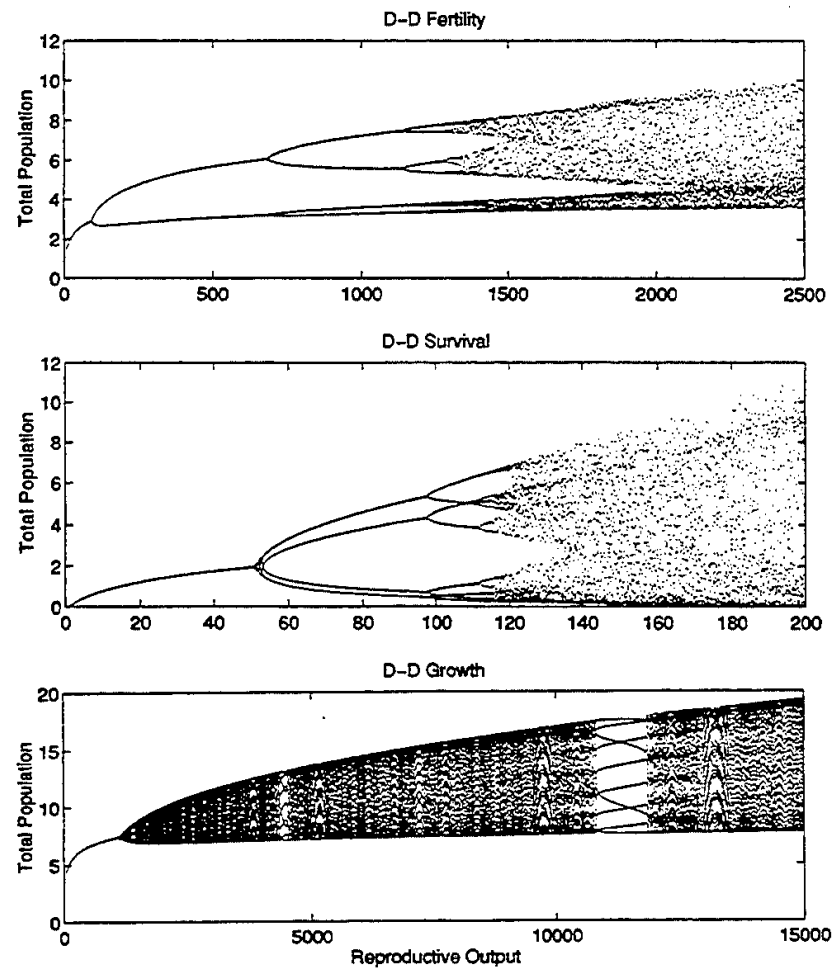

FIGURE 11. Bifurcation plots beyond the first bifurcation for the three two-stage examples, showing the long-term behavior of total population size $\left(N=n_{1}+n_{2}\right)$ as a function of reproductive output $\left(F_{2}\right.$ or, for the model with density-dependent fertility, $\left.f_{0}\right)$. Other parameters as in Figure 3.

Frequency locking is followed by more quasiperiodicity. Eventually, the invariant circle may begin to break up, or become wrinkled or folded, leading to chaotic dynamics.

Both patterns can be found in our examples. Figure 11 shows the bifurcation diagrams for the three models as reproductive output is increased even further, and Figure 12 shows examples of the attractors in the chaotic regime. Note that although all three models eventually become chaotic, the structures of the resulting attractors are quite different. 

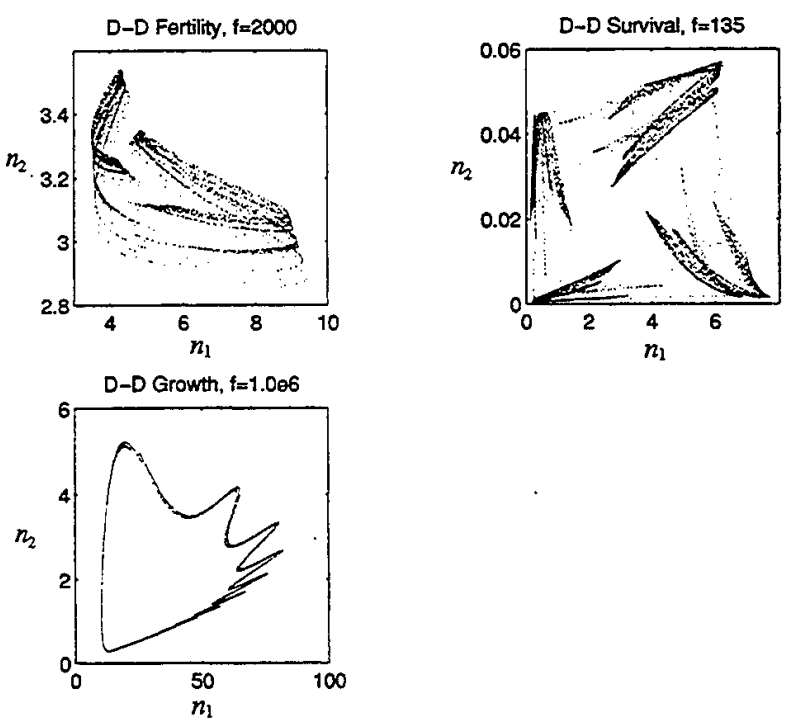

FIGURE 12. Phase portraits for chaotic attractors for the three two-stage examples, plotting $n_{2}$ vs. $n_{1}$ for several thousand iterations, after transients have died out. For the growth plot, the vertical axis is $100,000 \times n_{2}$. Values of reproductive output ( $F_{2}$ or, for the model with density-dependent fertility, $f_{0}$ ) are shown; other parameters as in Figure 3.

\section{Conclusion}

This chapter introduces some of the most immediately useful methods for analyzing population dynamics with matrix models. In combination with Chapter 3 on stochastic models (Tuljapurkar), and Chapter 6 on density-dependent models (Cushing), it should provide the tools needed to begin the analysis of matrix models and to understand the relevant literature.

Remember that the objective of a matrix model (indeed, any structured-population model) is to describe the individual-level processes of development, growth, aging, survival, and reproduction to their population-level consequences. How much detail is required depends on the goals of the model. To characterize population response to a current environment, a linear, time-invariant model provides a powerful tool. To characterize the effects of population and environmental variability, the stochastic tools in Chapter 3 are necessary. To include feedback between the state of the population and the environmental conditions faced by the indi- 
viduals, incorporate density-dependent nonlinearities and use the methods presented here and in Chapter 6. Regardless of the level of detail in the model, the analysis usually considers long-term dynamics, problems of ergodicity, and a perturbation analysis.

\section{Acknowledgments}

Thanks to the students in the Summer School for their participation, and to Myriam Barbeau, Mark Hill, Mike Neubert, and Shripad Tuljapurkar for detailed comments on the manuscript. Financial support provided by National Science Foundation grants DEB-9211945 and OCE-9302874, and Office of Naval Research grant URIP N00014-92-J-1527. This is Woods Hole Oceanographic Institution Contribution 9224.

\section{Literature Cited}

Beddington, J. 1974. Age distribution and the stability of simple discrete time population models. Journal of Theoretical Biology 47: 65-74.

Brault, S., and H. Caswell. 1993. Pod-specific demography of killer whales (Orcinus orca). Ecology 74: 1444-1454.

Caswell, H. 1986. Life cycle models for plants. Lectures on Mathematics in the Life Sciences 18: 171-233.

- 1989a. Matrix Population Models: Construction, Analysis, and Interpretation. Sinauer, Sunderland, Mass.

- $1989 b$. The analysis of life table response experiments. I. Decomposition of treatment effects on population growth rate. Ecological Modelling 46: 221-237.

- 1989c. Life history strategies. Pp. 285-308 in J. M. Cherrett, ed., Ecological Concepts. Blackwell, Oxford, Engl.

- 1996a. Demography meets ecotoxicology: Untangling the population level effects of toxic substances. Pp. 255-292 in M. C. Newman and C. H. Jagoe, eds., Ecotoxicology: A Hierarchical Treatment. Lewis Publishers, Boca Raton, Fla.

- 1996b. Second derivatives of population growth rate: calculation and applications. Ecology 77: 870-879.

. In press. Analysis of life table response experiments. II. Alternative parameterizations for size- and stage-structured models. Ecological Modelling.

Caswell, H. and M. C. Trevisan. 1994. The sensitivity analysis of periodic matrix models. Ecology 75: 1299-1303.

Chapman, F.M. 1932. Handbook of Birds of Eastern North America. 2nd, rev. ed. D. Appleton, New York. 
Cushing, J. M. 1988. Nonlinear matrix models and population dynamics. Natural Resource Modeling 2: 539-580.

DeAngelis, D. L., L. J. Svoboda, S. W. Christensen, and D. S. Vaughan. 1980. Stability and return times of Leslie matrices with densitydependent survival: Applications to fish populations. Ecological Modelling 8: 149-163.

Dennis, B., R. A. Desharnais, J. M. Cushing, and R. F. Costantino. 1995. Nonlinear demographic dynamics: Mathematical models, statistical methods, and biological experiments. Ecological Monographs 65: 261281.

Getz, W. M., and R. G. Haight. 1989. Population Harvesting: Demographic Models of Fish, Forest, and Animal Resources. Princeton University Press, Princeton, N.J.

Goodman, L. A. 1967. On the reconciliation of mathematical theories of population growth. Journal of the Royal Statistical Society A 130: 541-553.

Guckenheimer, J., G. Oster, and A. Ipaktchi. 1977. The dynamics of density dependent population models. Journal of Mathematical Biology 4: 101-147.

Hale, J., and H. Koçak. 1991. Dynamics and Bifurcations. SpringerVerlag, New York.

Hartshorn, G. S. 1975. A matrix model of tree population dynamics. Pp. 41-51 in F. B. Golley and E. Medina, eds., Tropical Ecological Systems. Springer-Verlag, New York.

Horn, R. A., and C. A. Johnson. 1985. Matrix Analysis. Cambridge University Press.

Keyfitz, N. 1967. Reconciliation of population models: Matrix, integral equation and partial fraction. Journal of the Royal Statistical Society A 130: 61-83.

. 1968. Introduction to the mathematics of population. AddisonWesley, Reading, Mass.

Lefkovitch, L. P. 1965. The study of population growth in organisms grouped by stages. Biometrics 21: 1-18.

Leslie, P. H. 1945. On the use of matrices in certain population mathematics. Biometrika 33: 183-212.

- 1948. Some further notes on the use of matrices in population mathematics. Biometrika 35: 213-245.

Levin, S. A. 1981. Age-structure and stability in multiple-age spawning populations. Pp. 21-45 in T. L. Vincent and J. M. Skowronski, eds., Renewable Resource Management. Springer-Verlag, Heidelberg.

Logofet, D.O. 1993. Matrices and Graphs: Stability Problems in Mathematical Ecology. CRC Press, Boca Raton, Fla.

May, R. M. 1979. Bifurcations and dynamic complexity in ecological systems. Annals of the New York Academy of Sciences 316: 517-529. 
McDonald, D. B., and H. Caswell. 1993. Matrix methods for avian demography. Current Ornithology 10: 139-185.

Mesterton-Gibbons, M. 1993. Why demographic elasticities sum to one: A postscript to de Kroon et al. Ecology 74: 2467-2468.

Neubert, M. G., and M. Kot. 1992. The subcritical collapse of predator populations in discrete-time predator-prey models. Mathematical Biosciences 110: 45-66.

Sarukhán, J., and M. Gadgil. 1974. Studies on plant demography: $R a$ nunculus repens $\mathrm{L}$., $R$. bulbosus $\mathrm{L}$. and $R$. acris L. III. A mathematical model incorporating multiple modes of reproduction. Journal of Ecology 62: 921-936.

Seneta, E. 1981. Non-Negative Matrices and Markov Chains. 2nd ed. Springer-Verlag, New York.

Silva, J. A. L., and T. G. Hallam. 1992. Compensation and stability in nonlinear matrix models. Mathematical Biosciences 110: 67-101.

Werner, P. A., and H. Caswell. 1977. Population growth rates and age versus stage-distribution models for teasel (Dipsacus sylvestris Huds.). Ecology 58: 1103-1111.

Whitley, D. 1983. Discrete dynamical systems in dimensions one and two. Bulletin of the London Mathematical Society 15: 177-217.

Wiggins, S. 1990. Introduction to Applied Nonlinear Dynamical Systems and Chaos. Springer-Verlag, New York.

Wikan, A., and E. Mjolhus. 1995. Periodicity of 4 in age-structured population models with density-dependence. Joumal of Theoretical Biology 173: 109-119. 